\title{
Effects of storage load and word frequency on pupillary responses during short-term memory
}

ERROL L. ELSHTAIN AND THEODORE SCHAEFER, Jr., SCIENCE AND ENGINEERING, INC., Waltham, Mass. 02154

Letter-word memory sequences differing in word frequencies and average storage loads ( $A S L)$ were presented aurally to nine $S$ s. Pupil diameter, measured continuously and electronically with infrared light retroreflected from the retina, increased slightly (3.5\%) during loading; markedly during recall (9\% to $23 \%$ with increasing ASL). Word frequency affected pupil size only at the highest ASL. Recall errors, unaffected by word frequency, increased with increasing $A S L$. Contrasted with recall errors, pupil dilation indicated processing at lowest ASL ( $9 \%$ increase), some effect of word frequency, and a greater range of effect in relation to $A S L$.

Lloyd (1964) studied short-term memory as a function of item difficulty and average storage load (ASL). Recall errors increased with increasing item difficulty and larger ASL, but item difficulty was less important at higher ASLs.

The present experiment repeated part of Lloyd's to evaluate hypotheses by Hess \& Polt (1964) that pupil size correlates with problem difficulty (i.e., word frequency), and by Kahneman \& Beatty (1966) that pupil size indicates amount of material (i.e., ASL) being processed. It seemed likely that Lloyd's procedure could indicate whether pupil size varies with item difficulty, ASL, or both.

Method

Four female and five male Ss, 19 to 42 years old, most with advanced degrees, were individually presented with six taperecorded sequences of letter-word pairs. The sequences differed in item difficulty, i.e., high (H) or low (L) frequency words (100 or 0.22 per million, respectively, from Thorndike \& Lorge, 1944); and in ASL, i.e., an average of $2.5,4.5$, or 6.5 items stored before recall.

Each sequence contained 20 items and 10 recall points. Before recall, one, two, or three items had been presented for recall at that point, but ASL, the total number of preceding items, ranged from one to eight. Extra items were added towards the end of high ASL sequences to maintain required loads. These items were elicited after the tenth recall point, but not scored. See Lloyd (1964) for details.

Items were presented at a 4 -sec rate. A brief 500-cps tone $1 \mathrm{sec}$ before each recall point prepared $S$ for naming the word(s) associated with the ensuing letter cue. Sequences were presented in the order $\mathrm{H} \mathrm{2.5,} \mathrm{L} \mathrm{2.5,} \mathrm{L} \mathrm{4.5,} \mathrm{H} \mathrm{4.5,} \mathrm{H} \mathrm{6.5}$, and $\mathrm{L} \mathrm{6.5,} \mathrm{with}$ 2 - to 5 -min rests between sequences.

$S$ sat, looking through a hooded headrest into a $10 \times 15 \mathrm{~cm}$ dichroic mirror mounted $13 \mathrm{~cm}$ from $S$ 's eye, $45 \mathrm{deg}$ to line of sight, in the path of an infrared beam. In this mirror $S$ fixated a small black cross in a transilluminated $45 \times 64 \mathrm{~cm}$ homogeneous white field suspended $71 \mathrm{~cm}$ above the mirror. Baseline pupil diameter was adjusted to $3.5-4.5 \mathrm{~mm}$ by controlling fluorescent lamps illuminating the field.

Vertical and horizontal pupil diameter, vertical and horizontal eye movements, and $S-R$ events were displayed on a five-channel recorder using an electronic oculo-pupillometer. This instrument projects a collimated infrared beam into the eye and back through the pupil onto a star-tracker photocathode for continuous measurement of pupil size and eye movements.

E recorded recall errors (including omissions) directly on the record. Horizontal pupil diameter was scored for the following intervals: $P, 1$ sec preceding an item or recall tone; $M, 3 \mathrm{sec}$ following an item ( $2 \mathrm{sec}$ if the item preceded a tone); and $\mathrm{R}, 3 \mathrm{sec}$ during recall. For each interval the largest diameter was tabulated to the nearest $0.1 \mathrm{~mm}$.

Results

Figure 1 shows median recall errors and pupil diameters (mm), during $\mathrm{R}$, for $\mathrm{H}$ and $\mathrm{L}$ sequences as a function of ASL. For recall errors, the Kruskal-Wallis $\mathrm{H}$, one-way analysis of variance, was significant for the effect of ASL (H sequences, $p<.001 ; L$, $\mathrm{p}<.01)$. Mann-Whitney U-tests showed significantly different $(p<.02)$ error scores between ASLs within both $H$ and $L$ sequences, excepting $L 4.5$ and $L$ 6.5. Although more recall errors occurred for $\mathrm{L}$ than $\mathrm{H}$ sequences at ASL 4.5 and 6.5, U-tests of these differences were not significant.

Pupil size during $\mathbf{P}$ intervals did not vary appreciably or consistently over time or sequences. During $M$ there was an overall $3.5 \%$ increase (per cent change $=\mathrm{M}-\mathrm{P} / \mathrm{P} \times 100, \mathrm{M}$ and $\mathrm{P}$, average of individual means) with no trends over time or sequences. During $\mathrm{R}$, pupil diameter increased $14.9 \%$ (overall average) and differences between sequences were marked. Overall, pupil diameter averaged $3.60 \mathrm{~mm}$ during $P, 3.71$ during $M$, and 4.11 during $R$. Pupil dilation during $R$ increased with increasing ASL for both $\mathrm{H}$ and $\mathrm{L}$ sequences, but significantly only within $\mathrm{L}$ sequences (Kruskal-Wallis $\mathrm{H}, \mathrm{p}<.05$ ). Mann-Whitney U-tests showed significantly higher dilation during $R$ for sequence $L 6.5$ compared to $\mathrm{L} 2.5(\mathrm{p}<.05)$ or $\mathrm{L} 4.5(\mathrm{p}<.02)$. Pupil dilation during $R$ was higher in sequence $L 6.5$ than $H 6.5(p<.10)$, but there were no appreciable differences between $L$ and $H$ sequences at ASL 2.5 or 4.5 .

Discussion

In general, these results support both Hess \& Polt and Kahneman \& Beatty: Pupil diameter increased during memorization, and especially during recall. Item difficulty and storage load both influenced magnitude of dilation during recall. The greater dilation for low-frequency words at the highest storage load suggests that recall is more difficult for low-frequency words. Although error scores were comparable for both word frequencies at all storage loads, pupillary responses may indicate more "work" in attempting to recall low-frequency words. This would agree with reports by Hess \& Polt (1964) and Schaefer et al (1968) that pupil dilation increases with increased difficulty of multiplication problems.

Lloyd (1964) found that recall errors were greater for lowfrequency words, especially at low storage loads. Error scores in this study were comparable to Lloyd's except that there were no appreciable differences between high- and low-frequency sequences at any storage level. Since sequence $H 2.5$ was first for all Ss in this study, Lloyd's word frequency effect at low storage load may have been masked. Also, three Ss stated a preference for

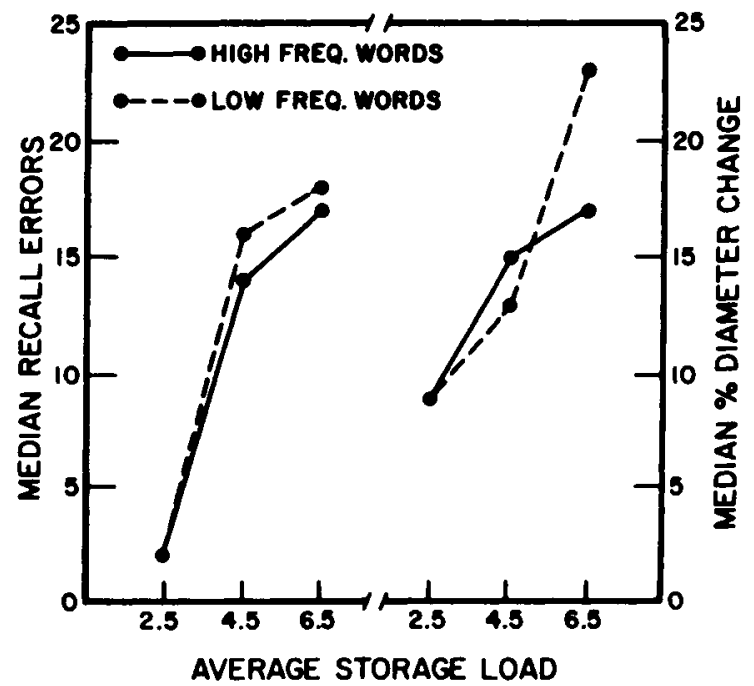

Fig. 1. Median recall errors (left) and median per cent dilation (right) for high-and low-frequency words at each average storage load. 
remembering the unusual, low-frequency words, whereas Lloyd's Ss were chosen from Ss who practiced with high-frequency sequences.

Compared to recall errors, pupil size during recall seems a better indicant of memory processing. Dilation (median $9 \%$ ) occurs even at ASL 2.5 where errors are nearly zero, and dilation continues to increase over a greater range than error scores which are limited to total words for recall.

The task complexity (e.g., recall load and ASL were confounded during memory and recall) of the present study may have masked several pupillary effects. For example, Kahneman \& Beatty, presenting one item per second, report an almost stepwise increase in dilation as memory load increases. In the present study the 3-sec interval between items may have been too long to sustain pupillary increase across items. Ss' attempts to organize the material may have also interfered with dilation: Four Ss tried to guess which letter would be the next recall cue, memorizing only those words associated with that letter. This tactic resulted in few errors at ASL 2.5, but poor success at higher ASLs. Thus, interacting effects of recall load and ASL, or limited storage at higher ASLs may differentially affect dilation during recall.

Pupillometry has a potential value in the study of learning, memory, and problem solving, but future experiments should use simpler memory tasks in which, for example, recall load, storage load, and problem solving strategies are less confounded.

\section{REFERENCES}

HESS, E. H., \& POLT, J. M. Pupil size in relation to mental activity during simple problem-solving. Science, 1964, 143, $1190-1192$.

KAHNEMAN, D., \& BEATTY, J. Pupil diameter and load on memory. Science, 1966, 154, 1583-1585.

LLOYD, K. E. Short-term retention as a function of word frequency. $J$. verbal Leam. verbal Behav., 1964, 3, 286-289.

SCHAEFER, T., Jr., FERGUSON, J. B., KLEIN, J. A., \& RAWSON, t. B. Pupillary responses during mental activities. Psychon. Sci, 1968, $12,137-138$

THORNDIKE, E. G., \& LORGE, I. The teacher's word book of 30,000 words. New York: Columbia University Press, 1944. 